ISSN Impreso: 1794-9920

ISSN Electrónico: 2500-9338

Volumen 17- $\mathrm{N}^{\circ} 2$

Año 2017

Págs. 81-90

\title{
AVANCES EN EL ANÁLISIS DEL ESTRÉS LABORAL A PARTIR DE LA CAPTURA DE NEUROSEÑALES EN EL CLIENTE INTERNO DENTRO DE UNA ORGANIZACIÓN TEXTIL EN LA CIUDAD DE PAMPLONA.*
}

\author{
Luz Ángela Moreno Cueva* \\ Enlace ORCID: http://orcid.org/0000-0002-3212-7087 \\ Marisol Maestre Delgado ** \\ Enlace ORCID: http://orcid.org/0000-0002-0548-2710 \\ Ludy Amira Flórez Montañez *** \\ Enlace ORCID: http://orcid.org/0000-0001-5345-9710 \\ Luis Manuel Palomino Méndez **** \\ Enlace ORCID: http://orcid.org/0000-0002-1632-2800 \\ Juan Manuel Villamizar Ramírez ${ }^{* * * *}$ \\ Enlace ORCID: http://orcid.org/0000-0002-7733-0927
}

Fecha de Recepción: 12 diciembre 2016

Fecha de Aprobación: 1 marzo 2017

\section{Resumen:}

En este artículo se presenta un estudio de caso del análisis de neuroseñales de un cliente interno de una organización de tejidos en la ciudad de Pamplona, ante situaciones cotidianas con el fin de evaluar cómo las condiciones laborales afectan el desempeño de los trabajadores en relación a su rendimiento laboral, siendo el estrés un problema que acoge a la organización, causando disminución en la productividad y en la competitividad, por ende en la disminución de utilidades. En la investigación se utilizó un instrumento base denominado Emotiv Insight, con el cual se realizaron las mediciones de las frecuencias emitidas por el cerebro. Al ejecutar los eventos que englobaban el experimento, se pudo observar que al cliente interno cuando le asignan una tarea que no le agrada tiene alto nivel de estrés, mientras que al hacerle entrega de un incentivo los niveles del mismo estado comienzan a decaer. A manera de conclusiones se puede decir que el estrés se ve reflejado cuando: se dan órdenes bruscas; el gerente se encuentra al lado del trabajador mientras labora, así como en los momentos en el que el jefe genera presión; y por último, que los incentivos son un eje fundamental en la organización.

Palabras Claves: Estrés, neuroseñales, organizaciones, cliente interno, emociones, tejidos.

\footnotetext{
* Administradora Comercial y de Sistemas-Magister en Dirección y Administración de Empresas MBA-Luz.moreno@unipamplona.edu.co

**Economista-Magister en Educación-Mmaestre24@hotmail.com

***Licenciada de Comercio-Administradora de Empresas-Especialista en Pedagogía Universitaria--ludyfm@unipamplona.edu.co

${ }^{* \star * \star A d m i n i s t r a d o r ~ d e ~ E m p r e s a s-M B A ~ e n ~ A d m i n i s t r a c i o ́ n-l u i s m a n u e l @ u n i p a m p l o n a . e d u . c o ~}$
} 
${ }^{* * \star \star *}$ Contador Público. - Especialista y Magíster en Finanzas- Docente Universidad de Pamplona Correo electrónico: juanmvillamizar@hotmail.com, juanmanuel@unipamplona.edu.co

\title{
ADVANCES IN THE ANALYSIS OF LABOR STRESS FROM NEURO-SIGNALS CAPTURED IN THE INTERNAL CLIENT WITHIN A TEXTILE ORGANIZATION IN THE CITY OF PAMPLONA
}

\begin{abstract}
This paper presents a case study of the neurosensal analysis of an internal client of a tissue organization in the city of Pamplona, in face of everyday situations in order to evaluate how the working conditions affect the performance of workers in relation to their Work performance, stress being a problem that welcomes the organization, causing a decrease in productivity and competitiveness, hence in the decrease of profits. The research used a base instrument called Emotiv Insight, which was used to measure the frequencies emitted by the brain. When executing the events that included the experiment, it was observed that the internal client when assigned a task that does not like it has a high level of stress, while giving an incentive the levels of the same state begin to decline. By way of conclusions we can say that stress is reflected when: abrupt orders are given; The manager is next to the worker while working, as well as in the moments in which the boss generates pressure; And last but not least, that incentives are a fundamental axis in the organization
\end{abstract}

Keywords: Stress, neuro-signals, organizations, internal client, emotions, tissues.

\section{AVANÇOS NA ANÁLISE DO ESTRESSE TRABALHADOR DE NEURO-SINAIS CAPTURADOS NO CLIENTE INTERNO DENTRO DE UMA ORGANIZAÇÃO TÊXTIL NA CIDADE DE PAMPLONA}

\begin{abstract}
Resumo
Este artigo apresenta uma análise de estudo de caso de sinais neurais de um cliente interno de um tecido organização na cidade de Pamplona é apresentado para situações cotidianas, a fim de avaliar como as condições de trabalho afetam o desempenho do trabalhador em relação ao seu desempenho no trabalho, o stress ser um problema que saúda os esforços a empreender, causando diminuição da produtividade e da competitividade, portanto, os lucros em declínio. Foi usado um instrumento chamado base Emotiv Insight, com a qual as medições das frequências emitidas pelo cérebro foram realizadas em investigação. Quando você executa os eventos que englobava o experimento, observou-se que o cliente interno quando atribuída uma tarefa que não gostaria de ter altos níveis de estresse, enquanto ao presenteá-lo com um incentivo níveis mesmo estado começará a declinar. A título de conclusão, podemos dizer que o estresse é refletida quando: ordens repentinas são dadas; o gerente é ao lado do trabalhador enquanto ele trabalha, e nos momentos em que o chefe coloca pressão; e útlimo, que os incentivos são uma pedra angular na organização.
\end{abstract}

Palavras-chave Stress, neuro-sinais, organizações, cliente interno, emoções, tecidos. 


\section{AVANCES EN EL ANÁLISIS DEL ESTRÉS LABORAL A PARTIR DE LA CAPTURA DE NEUROSEÑALES EN EL CLIENTE INTERNO DENTRO DE UNA ORGANIZACIÓN TEXTIL EN LA CIUDAD DE PAMPLONA \\ Luz Ángela Moreno Cueva- Marisol Maestre Delgado -Ludy Amira Flórez Montañez- Luis Manuel Palomino Méndez}

\section{INTRODUCCIÓN:}

Una persona realiza mejor su trabajo cuando se encuentra cómoda, entendiéndose esto como el estado emocional en que está feliz con la tarea que desempeña en una empresa.

Hoy día se tiene evidencia que existe un gran número de enfermedades que tienen como origen el estrés en las personas, esto hace reflexionar a empresarios y trabajadores en qué actividades es necesario desarrollar para obtener beneficios sociales y económicos. En éste trabajo se aborda el estudio de las actividades que se realizan de forma cotidiana en una empresa con el fin de clasificarlas si son buenas o malas prácticas para que los trabajadores se encuentren en condiciones emocionales óptimas.

En el desarrollo del conocimiento la multidisciplinareidad se ha convertido en la gran posibilidad de abordar un fenómeno desde varios saberes que en los procesos metodológicos se complementan.

Uno de estos saberes que se está acoplando al desarrollo organizacional es la evolución de la neurociencia. Tomando cada día mayor relevancia en todo lo relacionado a medir o conocer aptitudes, estados de ánimos en los que los clientes internos están inmersos afectando directamente la evolución empresarial de una determinada organización. Su aplicación se ve claramente en el marketing y en todo el entorno de la organización. Dado la importancia de los resultados se está extrapolando hacia diversos campos que incluyen principalmente la educación, herramientas tecnológicas y medios de comunicación, sobre todo en lo que tiene que ver, con las redes sociales

En el mismo sentido en la primera parte del trabajo se explica brevemente lo que respecta al marco teórico, mostrando la relación entre las distintas áreas que se están abordando en el experimento; seguidamente la metodología utilizada así como la ejecución de los procedimientos que se tuvieron en cuenta para el desarrollo experimental y finalmente los resultados y las conclusiones, que son el fin del estudio.

\section{MARCO TEÓRICO:}

La gerencia actual tiene como enfoque estratégico el crear Ventajas competitivas en sus productos o servicios que permitan sostenerse y mejorar la perspectiva en el contexto mediante el posicionamiento y penetración de nuevos mercados. Porter 1991, economista y pionero irrefutable en
Temas de competitividad empresarial, expone en su libro "La ventaja competitiva de las naciones" que la estrategia competitiva constituye el éxito o fracaso de las empresas. Considera que toda habilidad o destreza se convierte en una estrategia, y al hacer uso de esa destreza mide la capacidad de competir frente al mercado, la competencia, y el posicionamiento de sus productos y/o servicios. La mayor contribución es el diamante de la competitividad donde Porter expone que el centro de la competencia radica en el grado de innovación que se tenga a nivel empresarial la cual da pauta a determinar: factores de oferta, integración con empresas relacionadas, conocimiento del mercado, estrategia empresarial, estructura y competencia, oportunidades y apoyos institucionales y/o públicos.

En este orden de ideas se reconoce que la competitividad se construye desde la misma persona, si este es competitivo en un escenario laboral globalizado la organización puede llegar ser competitiva. Esto nos lleva que el capital humano dentro de una organización es considerado el motor que dinamiza el quehacer de la empresa mediante la motivación, el empoderamiento, el liderazgo, perfil idóneo, apostándole al incremento de la productividad y la marca de diferenciación frente a la competencia. Así mismo conviene precisar que la implementación de estrategias de talento humano permite el desarrollo eficiente de los diferentes roles donde prima la calidad de procesos y productos o servicios. Aragón Sánchez, et al, 2004 en definitiva prima la necesidad de considerar al ser humano como el activo más importante al que hay que cuidar y motivar logrando la eficiencia y eficacia en busca del éxito empresarial.

Lo anterior conduce a que "Las organizaciones han modificado las prácticas gerenciales. En vez de invertir directamente en los productos y servicios, están invirtiendo en las personas que lo conocen y saben cómo crearlos, desarrollarlos y mejorarlos" (Chiavenato, 2008) lo anterior no tendría sentido si no se complementa con el desarrollo y perfeccionamiento de Planeación, Organización, Dirección y Control encaminados a la satisfacción entre los individuos que en ella confluyen, basados en un eficaz sistema de información interna y externa que le permita estar a la vanguardia de los cambios en el mercado.

Aquí conviene detenerse un momento a analizar qué toda esta dinámica ha generado escenarios competitivos de alianzas estratégicas, exigencia de mayores competencias en el ser humano y un desarrollo vertiginoso en la 
tecnología originando demanda de productividad, estudio de comportamiento del consumidor, disminución de costos suscitando sobrecarga laboral, mayores exigencias, agotamiento físico 0 mental, ambigüedad del rol, inestabilidad laboral, conflictos en el entorno, conllevando al estrés laboral o estrés es el trabajo. Ante esta dinámica prevalece la importancia de la calidad de vida en el trabajo (CVT) la cual depende del grado de satisfacción de las personas en relación con la empresa y el ambiente laboral.

Para ilustrar mejor lo anteriormente expuesto podemos hablar del síndrome de "Burnout" que traducido al castellano significa "estar o sentirse quemado, agotado, sobrecargado, exhausto" como resultado a esto podemos hablar de estrés laboral entendiéndose como cansancio emocional, perdida de motivación laboral, baja productividad, baja autoestima, lo que supone un coste personal, organizacional y social. La Organización Internacional del Trabajo (OIT), define el estrés laboral "enfermedad peligrosa para las economías industrializadas y en vías de desarrollo; perjudicando a la producción, al afectar a la salud física y mental de los trabajadores". La OIT determina como factores de riesgos: "las interacciones entre el contenido, la organización y la gestión del trabajo y las condiciones ambientales, por un lado, y las funciones y necesidades de los trabajadores, por otro lado, la OIT en (1998), menciona que "las condiciones que conducen al estrés en el trabajo y a otros problemas conexos de salud y seguridad" como las "condiciones normalmente denominadas factores psicosociales". Lo anterior permite que se establezca una clasificación en estos Factores Psicosociales: riesgos del medio ambiente físico, riesgos asociados al diseño de la tarea, organización del tiempo de trabajo, modalidad de la gestión Aragón Sánchez, Antonio; García Tenorio et al., 2004. Según la II Encuesta de Seguridad y Salud en el Trabajo, presentada por el Ministerio de Trabajo, en Colombia (2014) Concluye "las situaciones que mayor estrés generan en los trabajadores: el trabajo excesivamente exigente, falta de tiempo para completar las tareas, falta de claridad sobre la función del trabajador, desajustes entre las exigencias del trabajo y la competencia del trabajador". Adicionalmente otras situaciones como la falta de apoyo de la dirección, el acoso psicosocial, mal diseño de puesto de trabajo, escasas recompensas, ausencia ascensos, comunicación deficiente y una no eficiente gestión del cambio, contribuyen a un estrés laboral, (Seguridad \& Trabajo, 2014) Sin embargo, también hay factores relacionados como falta de influencia en el modo en que se lleva a cabo el trabajo solo, sobre todo si hace cara al público y a clientes, y/o exponerse a la violencia de un tercero recibiendo agresiones verbales.

En este mismo sentido Gabel-Shemueli, Rachel et al, (2012) en su trabajo "Estrés laboral: relaciones con inteligencia emocional, factores demográficos y ocupacionales" correlaciona el estrés laboral en 223 trabajadores, desde los constructos, dimensiones y subdimensiones, donde se evidencia que a mayor inteligencia emocional menor estrés laboral y profesional sin omitir que en los cargos de alta dirección se presenta mayor estrés. La manera como el individuo asume el estrés genera consecuencias en la competitividad de las empresas partir de la efectividad y eficiencia en las labores que realiza el trabajador.

Atendiendo a lo anteriormente mencionado, se concluye que el estrés es una causa fundamental en el desarrollo de las labores que tiene cada uno de los trabajadores, por lo tanto, conocer el origen de lo que lo provoca permite mitigar el problema, que al final se ven representados en la disminución de las utilidades de la empresa.

Anteriormente, la mejor forma de evaluar o cuestionar las opiniones, emociones y sensaciones de una persona era realizando encuestas, las cuales no eran una apropiada herramienta puesto que no siempre se dan respuesta con la mayor sinceridad del caso, incluso son varias situaciones las que afectan las respuestas de ellas, tales como el nivel de cansancio, la ocupación que tenga en el momento, miedo debido a las represarías que pueda tomar la persona que lo dirige, por lo que la encuesta es "una fotografía del instante en el cual se aplicó" (María \& Poncela, 2014) así mismo las autoras mencionan que en ocasiones no se tienen resultados certeros, pues existe manipulación por parte de los encuestadores o incluso beneficios que se les otorgaría a quienes la ejecutan. Es por ello que al verse alterados los resultados, en ocasiones las soluciones que se plantean no resultan efectivas debido a la falsa información que se representa en el documento.

Por ello, existen varias personas tales como Néstor Baidot ¿Por qué tus clientes se acuestan con el otro si dicen que les gusta tú? (Neuromarketing); Gustavo Vásquez con Neurociencia. Bases y fundamentos; Arhur Guyton autor de Anatomía y fisiología del Sistema Nervioso: Neurociencia Básica; Joseph LeDoux del libro El cerebro emocional; entre otros (Neurociencia et al., 2011) que han dedicado gran parte de sus vidas a estudiar el cerebro humano, y las relaciones que existen con él, dado que todas las acciones y emociones que se forman allí están dadas bajos impulsos eléctricos, por lo que ciertos empresarios como Tan Le, quien es la fundadora y CEO de la empresa Emotiv han estudiado y diseñado instrumentos que permiten capturar éstas señales, a través de las lecturas encefalografías "que permiten realizar una medición directa, de las señales eléctricas del cerebro ante diferentes estímulos" (Demoscopia, 2013), teniendo en cuenta que dependiendo de la parte donde se ubique el movimiento cerebral se deduce la emoción que se está presentado. Ésta acción sólo se podía realizar a través de instrumentos médicos como las lecturas electroencefalográficas, siendo algo complejas sobre todo cuando se necesitaban hacer 


\section{AVANCES EN EL ANÁLISIS DEL ESTRÉS LABORAL A PARTIR DE LA CAPTURA DE NEUROSEÑALES EN EL CLIENTE INTERNO DENTRO DE UNA ORGANIZACIÓN TEXTIL EN LA CIUDAD DE PAMPLONA}

Luz Ángela Moreno Cueva- Marisol Maestre Delgado -Ludy Amira Flórez Montañez- Luis Manuel Palomino Méndez

mediciones para observar al consumidor ante un evento donde él no podía estar totalmente quieto.

Con el paso del tiempo se han desarrollado varios dispositivos de lecturas electroencefalograficas de bajo costo, como el Emotiv Epoc, que fue la primera versión desarrollada por la empresa, con el se puede capturar diferentes emociones, expresiones faciales e incluso manipular ciertos dispositivos tales como una silla de rueda tan sólo con pensarlo siendo posible gracias a 14 sensores como se puede apreciar en la Figura 1 que debían humedecerse para que existiera la conductividad.

Figura 1. Dispositivo Emotiv EPOC

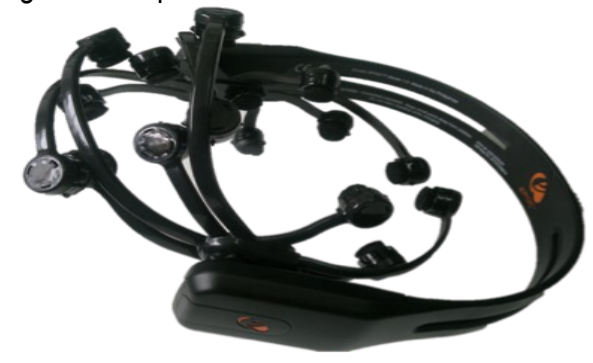

Fuente: Elaboración propia

Para evitar tantas molestias en el usuario la misma empresa creo el dispositivo Emotiv Insgiht que se observa en la figura 2 con cinco sensores de polímero que por su composición química no requiere que se humedezcan los sensores, capturando ciertas emociones y acciones que tiene una persona en determinado momento.

Figura 2. Dispositivo Emotiv Insight

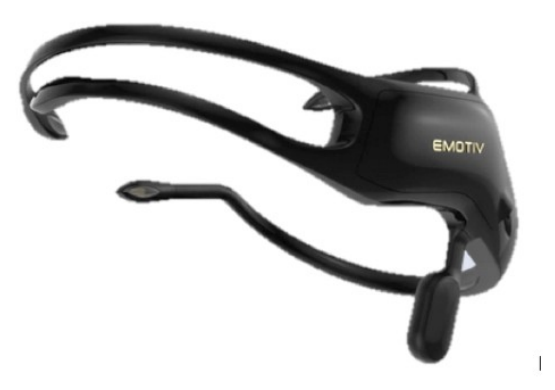

\section{Elaboración: Propia}

Con el paso del tiempo las grandes empresas han comenzado a involucrarse con ellos para conocer de forma biológica a los diversos consumidores, de tal forma que se puede observar qué tan emotivo se encuentra una persona y cómo es su comportamiento mientras ejecuta la acción de una compra ó simplemente mientras observa algún tipo de material multimedia donde se publicite alguna marca (Moreno Cueva, Peña Cortés, \& González Sepúlveda, 2014) permitiendo hacer correcciones tempranas en los momentos exactos donde se tiene menor atención por parte del usuario.

Hoy día existen varias organizaciones que se han encargado de llevar esta tecnología a las personas 0 empresas pequeñas, permitiendo que tengan disponible herramientas de bajo costo.

Para efectuar el trabajo del presente artículo se utilizó uno de los dispositivos que oferta la empresa australiana Emotiv, siendo este el Insight. Éste artefacto lee, captura y procesa las señales eléctricas del cerebro a través de sus cinco (5) sensores de polímero hidrófilo, haciendo énfasis en seis (6) métricas enfoque, estrés, emoción, relajación, interés y compromiso tanto a largo como a corto plazo, un ejemplo se muestra en la figura 3, lográndolo de la lectura de la corteza frontal, la parietal-temporal y la parietaloccipital (Science, 2017).

Figura 3. Interfaz de captura de neuroseñales de Emotiv

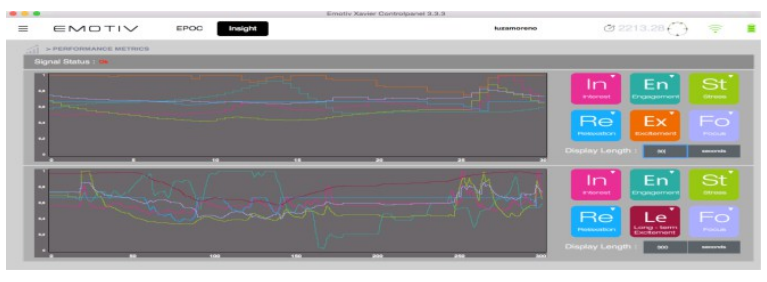

\section{METODOLOGÍA:}

Para efectuar la investigación se utilizó un enfoque mixto con preponderancia cualitativa, en donde se aplicaron diferentes tipos de herramientas para la recolección de la información.

La información tipo cualitativa se pudo recolectar a partir del diligenciamiento de las encuestas aplicadas a los involucrados en el experimento. Mientras que la cuantitativa fue obtenida de la utilización del dispositivo Emotiv Insight.

Antes de iniciar con el experimento se determinó una metodología que permitió un desarrollo ordenado, dada por 6 momentos. 
Figura 4. Metodología implementada para el desarrollo de los experimentos

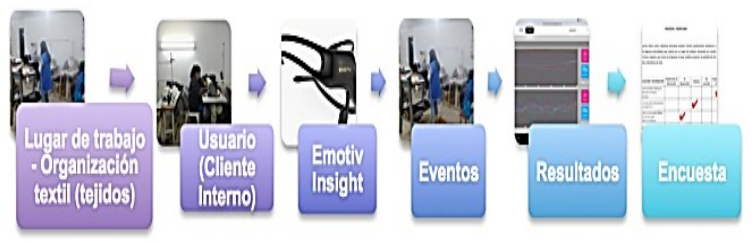

Fuente: Elaboración propia

Inicialmente se hizo un reconocimiento del lugar, analizando los espacios en los que el usuario iba a laborar, así como también el escenario donde los investigadores se iban a ubicar para no interferir en su visual y de ésta forma desligar su comportamiento en la medida de lo posible. En cuanto a los compañeros de trabajo jefes y demás se estableció previamente una reunión donde se les explicaba los parámetros del experimento y se le daban las pautas de las intervenciones que debían realizar cada uno de ellos respecto al cliente interno.

Posteriormente se hizo la instalación de los equipos con las debidas explicaciones y autorizaciones previamente mencionadas, asegurándose que el usuario estuviese realizando buen contacto con los sensores de la interfaz cerebro computador. Se realizó un entrenamiento que constaba de una fase con ojos abiertos y otra con los ojos cerrados para mejorar la percepción de las neuroseñales en el usuario en particular.

En cuarto lugar, se inició la captura de la información por medio del Emotiv Insight posterior al tiempo inicial de exaltación del usuario por el uso de los equipos (tiempo mientras olvida que tiene puesto el equipo la persona). Durante el tiempo de captura y registro de las neuroseñales se llevaban a cabo los eventos mencionados en la sección previa.

Por último se realiza una valoración cuantitativa al interpretar numéricamente los valores de las señales obtenidas y de igual forma una valoración cualitativa por medio de una encuesta.

Una vez se obtiene la captura de las neuroseñales, se analizan las variables respecto al tiempo en el que surgió cada evento con el fin de entregar resultados a los empresarios.

Ahora bien, antes de iniciar el experimento se revisaron aquellas limitaciones que poseía el dispositivo tales como: el alcance que se tenía entre la computadora y el Emotiv
Insight el cual era de cuatro (4) metros; no todas las personas se pueden evaluar pues el tamaño de la cabeza es importante, teniendo presente que todos los sensores del dispositivo deben estar en contacto con el cuero cabelludo; y por último se requiere de conexión a Internet, sin embargo se puede mencionar que existe un alto porcentaje de funcionalidad y certeza (Science, 2017).

Posteriormente se ubicaron las distintas empresas en la ciudad de Pamplona, que tienen como objeto social la producción de tejidos (textil) de tal manera que se generó una base de datos de ellas. Seguidamente se mantuvo una conversación con el accionista mayoritario, a quien se le explicaba el objetivo de la investigación y cómo se llevaría acabó así como la entrega del consentimiento informado para efectos de legalidad (permisos) que ya han sido firmados.

La empresa donde se ejecutó el experimento tiene cuatro (4) clientes internos (trabajadoras), a las cuales se les informó los procedimientos que se realizaría. Una vez enteradas y aprobado el permiso por cada una de ellas, se dispuso a seleccionar a la persona más idónea para colocarle el dispositivo atendiendo a las limitantes anteriormente mencionadas.

El experimento se ejecutó en una (1) hora con diez (10) minutos, es decir, un total de 70 minutos, en los cuales se generaron cinco (4) eventos con el fin de evaluar las variación en sus emociones, principalmente en el estrés.

Con el fin de acostumbrar al usuario al dispositivo y evitar datos erróneos producidos por la evaluación se destinó un lapso como periodo de relajación del usuario, se comenzó a registrar las señales emitidas a partir del minuto 18 desde que el individuo hacia uso del aparato. Se escogió este momento por medio de la percepción del grupo investigador dado que las señales del sujeto se estabilizaron y se observó una mayor concentración en su trabajo.

Las acciones 0 eventos que se generaron para la evaluación fueron: cambio de la tarea normal en ejecución por una inusual, donde la gerente dio la orden que comenzara a cocer el cuello de un buso cuando ella se encontraba en sus tareas cotidianas. Seguidamente y como segundo evento la crítica, donde se tomaron acciones para que la compañera de trabajo experta en cuellos le indicara que no estaba quedando bien la elaboración del mismo. En tercer lugar el evento correspondiente a la presión, donde la Jefe y la compañera comenzaron a forzar a la trabajadora para que terminara rápido la costura que estaba a su cargo (culminación de la elaboración del cuello de un buso). Por último se quiso observar cómo reacciona la persona cuando se le hace 


\section{AVANCES EN EL ANÁLISIS DEL ESTRÉS LABORAL A PARTIR DE LA CAPTURA DE NEUROSEÑALES EN EL CLIENTE INTERNO DENTRO DE UNA ORGANIZACIÓN TEXTIL EN LA CIUDAD DE PAMPLONA}

Luz Ángela Moreno Cueva- Marisol Maestre Delgado -Ludy Amira Flórez Montañez- Luis Manuel Palomino Méndez

entrega de algún incentivo. En este caso se dio una entrada a cine por su labor efectuada ese día.

\section{RESULTADOS Y DISCUSION:}

En la presente sección, se ilustran los resultados correspondientes a la persona evaluada, siendo evidentes los cambios en sus emociones cada vez que estaba inmersa bajo uno de los eventos que se aplicaban conforme iba avanzando el experimento. En la figura $5 \mathrm{se}$ ilustra el entorno de trabajo donde se llevaron a cabo los experimentos, por motivos de confidencialidad se difuminó el rostro de los empleados y se mantendrá de forma anónima la empresa.

Figura 5. Entorno de trabajo donde se llevó a cabo los experimentos

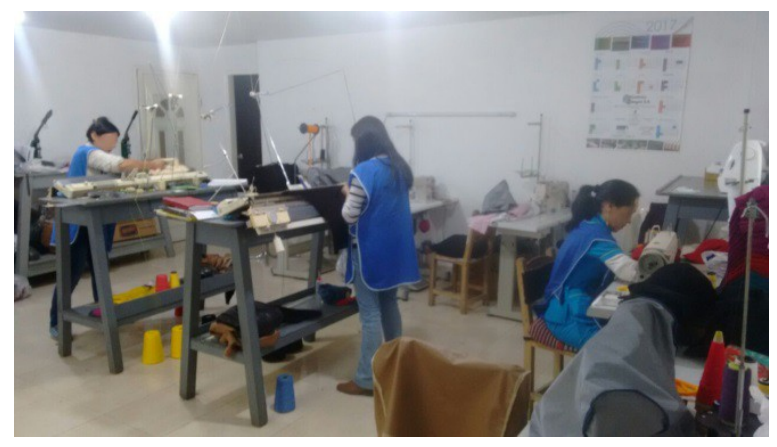

Fuente: Elaboración propia

Con el fin de dar una mayor claridad sobre el desarrollo de los experimentos se presenta la figura 6 donde se detalla una línea de tiempo en la que se aprecian los momentos en los cuales se aplican los eventos anteriormente mencionados. Ésta gráfica incluye los tiempos en segundos con el fin de contrastarlos con las figuras correspondientes al registro de las neuroseñales.

Con el fin de dar una mayor claridad sobre el desarrollo de los experimentos se presenta la figura 6 donde se detalla una línea de tiempo en la que se aprecian los momentos en los cuales se aplican los eventos anteriormente mencionados. Ésta gráfica incluye los tiempos en segundos con el fin de contrastarlos con las figuras correspondientes al registro de las neuroseñales.

Figura 6. Línea de tiempo de la generación de los eventos

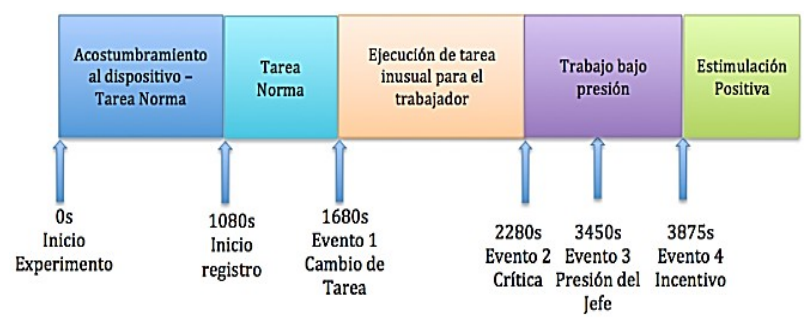

\section{Fuente: Elaboración propia}

En la figura 7 se puede apreciar un segmento del registro de las neuroseñales en etapa en la cual el operario realiza sus tareas de forma habitual, se aprecia claramente como la persona maneja niveles relativamente bajos de estrés y de una forma uniforme, producto de que el sujeto ya se acostumbró al dispositivo y que se encuentra realizando una tarea cotidiana, lo cual en psicología se puede denominar como la zona de confort del trabajador.

Figura 7. Estrés del operario al iniciarse el registro (minuto 18 del experimento)

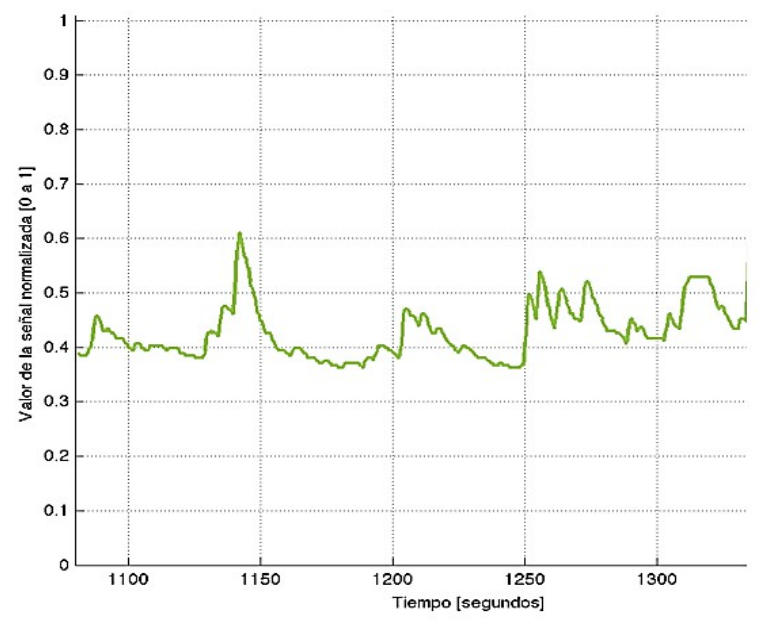

Fuente: Elaboración propia

Una vez la persona se encontraba estabilizada con el dispositivo y sus tareas cotidianas, las cuales eran elaborar las mangas de un buso, el gerente le ordena un cambio de 
procedimiento para elaborar una actividad completamente distinta (elaboración del cuello del buso) a la que ya venía trabajando, en la figura 8 se puede apreciar que su nivel de estrés era relativamente bajo hasta que el gerente le dio la nueva instrucción, llegando a los niveles más altos.

Figura 8. Estrés del operario al cambiarse la tarea a realizar por una no habitual

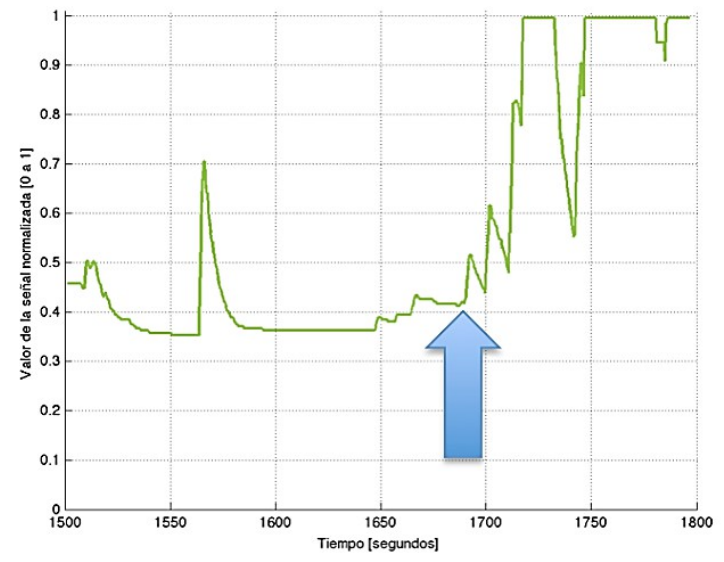

Fuente: Elaboración propia

La gerente de la empresa sólo da la orden y luego se aleja, a medida que la trabajadora toma practica comienza a bajar el nivel del estrés, aunque de una forma lenta. Tiempo después la compañera quien es experta en el la elaboración de cuellos, comienza a criticarla, comentándole que no está bien lo que está haciendo y que debería cambiar ciertas costuras, a lo que inmediatamente responde con nuevamente un incremento en la variable del estrés tal como se puede apreciar en la figura 9.

Figura 9. Estrés del operario ante la crítica de una compañera

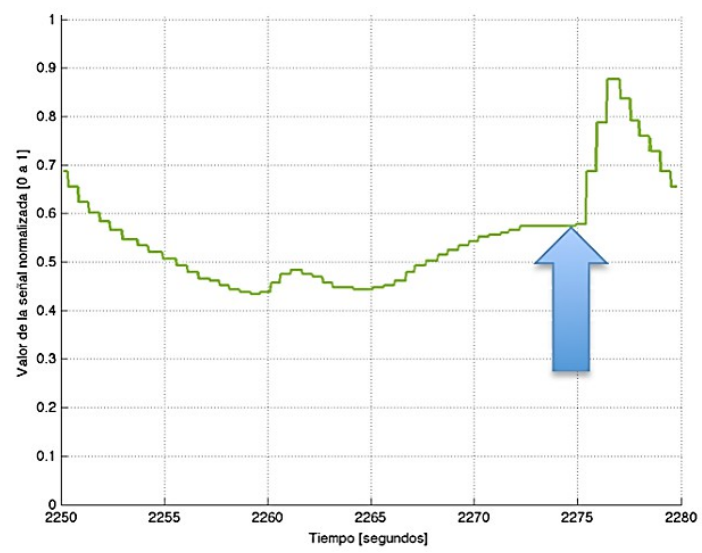

Fuente: Elaboración propia

La compañera se aleja del lugar y su nivel de estrés comienza a bajar y así se mantiene hasta que surge el siguiente evento el cual es la presión que genera el jefe al comenzar a preguntar si ya terminó el cuello que estaba cociendo, así como decirle que necesitan la terminación de

forma inmediata. Tal como se puede percibir en la figura 10, el estrés del operario subió a su máximo nivel, indicando que no se sentía agradado con la presión constante que efectuaba el jefe.

Figura 10. Estrés del operario ante la presión del jefe

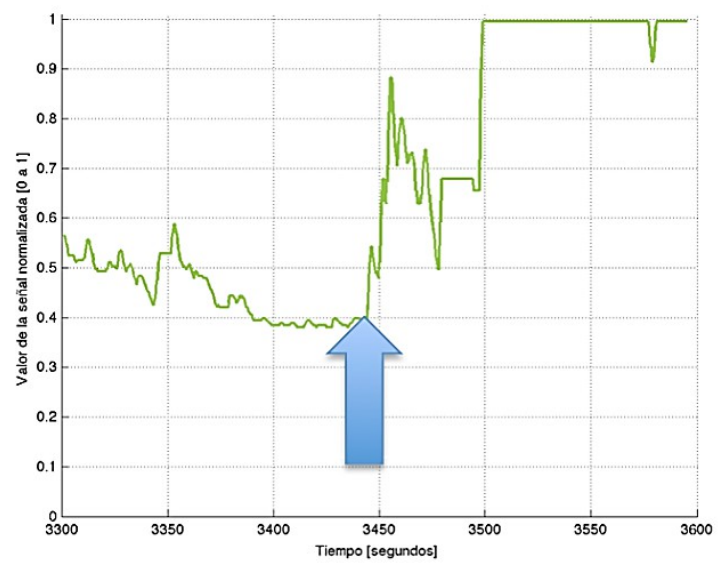

Fuente: Elaboración propia

Por último, una vez terminada la prenda se hizo una prueba de estimulación positiva al empleado por medio de la entrega de un incentivo, haciéndole saber que era la recompensa adicional a su pago por la actividad extenuante que realizó, lo que se ve representado en una drástica disminución del estrés como se muestra en la figura 11. Lo que permite al empresario comprender de forma soportada que se debe estimular a los trabajadores cuando son expuestos a altas presiones diferentes a las de su rutina de trabajo que se presenta día tras día.

Figura 11. Estrés del operario ante el incentivo 


\section{AVANCES EN EL ANÁLISIS DEL ESTRÉS LABORAL A PARTIR DE LA CAPTURA DE NEUROSEÑALES EN EL CLIENTE INTERNO DENTRO DE UNA ORGANIZACIÓN TEXTIL EN LA CIUDAD DE PAMPLONA \\ Luz Ángela Moreno Cueva- Marisol Maestre Delgado -Ludy Amira Flórez Montañez- Luis Manuel Palomino Méndez}

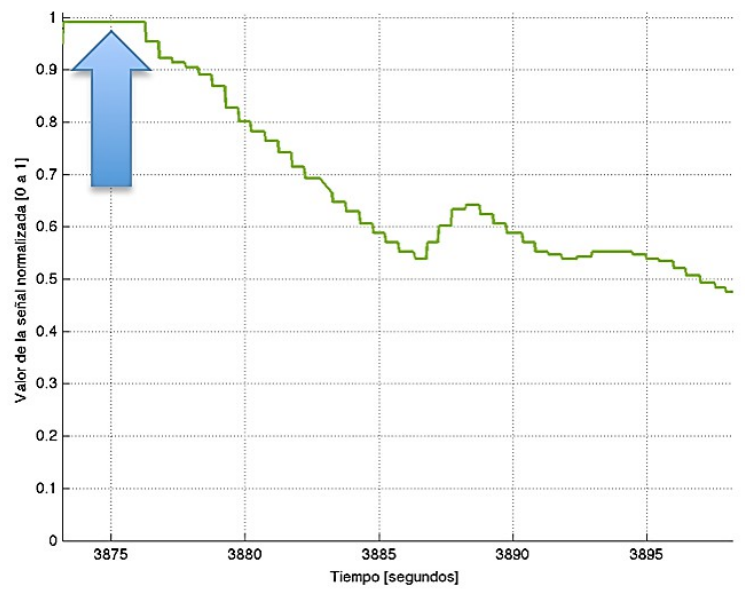

Fuente: Elaboración propia

Según se pudo apreciar en los resultados arrojados por la interfaz cerebro computador en cuanto al nivel estrés del cliente interno se evidencian cambios significativos para cada uno de los eventos recreados. Esto indica que se puede utilizar este tipo de experimentos como herramienta para evaluar actividades cotidianas que se llevan a cabo en la empresa y concluir si son buenas o malas prácticas.

\section{CONCLUSION:}

Las empresas deben evaluar continuamente los niveles de estrés que generan las diversas actividades cotidianas que desarrollan los clientes internos, de tal forma que se generen soluciones motivacionales que permitan al trabajador elevar su ánimo y por tanto su productividad.

Se demostró que existen acciones pequeñas tales como una entrada a cine que permite al trabajador motivarse $y$ seguir trabajando mejor día tras día.

Para evitar mayor nivel de estrés, es recomendable que el operario se especialice en alguna actividad en particular, de tal forma que permite adquirir mayores destrezas, lo cual no sólo mejora la producción en tanto cantidad como calidad, sino también se mejora el ambiente laboral, pues se tendrían menos personas irritadas.

La presión que se genera en un cliente interno no permite que realice su labor agradablemente lo que conlleva a que la persona pueda tener mayores errores e incluso intimidarla a pesar que esté realizando su mejor esfuerzo. Un gerente puede tener la percepción que entre más se presione a los trabajadores mejores resultados se van a obtener lo cual puede ser un gran error. Sin embargo, esto podría ser una buena técnica para casos extremos donde los trabajadores no estén realizando sus funciones por pereza o temas similares.

Según los resultados obtenidos en cuanto a críticas y presiones se recomienda que para el operario evaluado, en la medida de lo posible las observaciones negativas se hagan por parte de compañeros y no directamente por los jefes, con el fin de disminuir los niveles de estrés, lo cual puede ser producto del nivel de confianza que se tiene con los mismos.

Los incentivos son fundamentales a la hora de motivar 0 ver momentos de felicidad en los clientes internos, sobre todo en los tiempos en los que la empresa tienen horarios de trabajo extenuantes, con el fin de que ellos vean estás épocas como oportunidad, en donde existirán mayores beneficios tanto para ellos como para la empresa y por lo mismo se generará alegría conduciendo a ambientes laborales óptimos.

Una conclusión muy importante es la alta probabilidad que se genere un efecto cascada en el cliente interno, si la empresa no ofrece unas condiciones favorables de desempeño laboral.

\section{REFERENCIAS:}

Aragon Sanchez, Antonio; Garcia Tenorio, J., Perez Rodriguez, M. J., Sabater Sanchez, Ramon; Sanchez Marin, Gregorio; Sanchez Quiros, I., \& Sanz Valle, R. (2004). Fundamentos de dirección y gestión de recursos humanos, 46 .

Chiavenato, I. (2008). Gestión del Talento Humano. (M.-H. E. SA, Ed.) (3rd ed.).

Chiavenato, I. (2011). Administración de Recursos Humanos. (M. G. Hill, Ed.) (Novena).

Demoscopia, T. N. S. (2013). ¿Qué puede aportar la neurociencia al marketing y a la investigación de mercados? Juventud, Neurociencia, Tecnología $Y$ Subjetividad, 103, 51-68.

Gallegos, W. L. A. (2012). Estrés laboral en trabajadores desde el enfoque de los sucesos vitales Occupational stress of workers analyzed from an approach to vital events. Revista Cubana de Salud Pública, 38(4), 525-535.

María, A., \& Poncela, F. (2014). La madre de las encuestas sobre la guerra de las encuestas 2012. Revista Mexicana de Opinión Pública, 17, 110-133. http://doi.org/10.1016/S1870-7300(14)70902-0 
Moreno Cueva, L. Á., Peña Cortés, C. A., \& González Sepúlveda, H. (2014). Integración de un sistema de neuroseñales para detectar expresiones en el análisis de material multimedia. Revista Facultad de Ingeniería, 24(38), 29-40.

Neurociencia, L., Central, S. N., Perif, S. N., Neurona, L., Am, L., Hipot, E., ... Sentimiento, E. (2011). El neuromarketing: Un análisis neurocientífico del comportamiento de los consumidores. Universidad de Palermo.

Porter, M. (1991). 1 Michael Porter, La Ventaja Competitiva de las Naciones (1990) Edición en español: Javier Vergara Editor SA, Bs.As., 1991. (J. V. Editor, Ed.) (1st ed.).

Rodríguez Carvajal, R., \& Hermosilla, S. D. R. (2011). Los procesos de estrés laboral y desgaste profesional (burnout): diferenciación, actualización y líneas de intervención. Medicina $Y$ Seguridad Del Trabajo, 57, 72-88.

Science, T. H. E. (2017). Open Your Mind to Next Generation Brainwe EMOTIV Insight Introduction Vid. Retrieved from https://www.emotiv.com/insight/ HARDWARE

Seguridad, D., \& Trabajo, M. De. (2014). Principales generadores de estrés laboral en Colombia, pp. 2-3.

Zulia, U., Rondan, P., Lozano, P., Amelia, R., Huarcaya, A., Zulia, U., ... Huarcaya, A. (2012). Estrés laboral : relaciones con inteligencia emocional, factores demográficos y ocupacionales. Revista Venezolana de Gerencia, 17, 271-290. 